\title{
Peritoneal Dialysis Induced Bilateral Blindness in a Patient with End Stage Renal Disease
}

\section{Ana Dvalishvili ${ }^{1 *}$, Tatsiana Imshenetskya ${ }^{2}$ and Khatuna Sagamelidze ${ }^{1,2}$}

${ }^{1}$ New Vision University, High Technology Medical Center, University Clinic, Tbilisli, Georgia

${ }^{2}$ Belarusian Medical Academy of Post Graduate Education, Minsk, Belarus

*Corresponding Author: Ana Dvalishvili, New Vision University, High Technology

Medical Center, University Clinic, Tbilisli, Georgia.

DOI: 10.31080/ASMS.2021.05.1058
Received: August 21, 2021

Published: October 07, 2021

(C) All rights are reserved by Ana Dvalishvili., et al.

\section{Abstract}

Background: Bilateral acute blindness is a rare eye complication in an adult patients undergoing peritoneal dialysis and in this cases mostly is associated with a severe uncontrolled systemic hypotension. Information about the incidence, severity, and course of arterial hypotension induced eye complication in adults receiving chronic peritoneal dialysis (CPD) is limited.

Case Report: A 51 -year-old female with End Stage Renal disease of unknown etiology undergoing peritoneal dialysis for the previous 3 years. She had a longstanding history of persistent hypotension which was fluctuated from $90 / 60 \mathrm{mmHg}$ to $60 / 40 \mathrm{mmHg}$. She was having several episodes of total visual loss (during past 5 days), which were recovering after the sessions of peritoneal dialysis. After the last episode, when her BP was 60/30, she totally loss her VA.

Results: Bilateral Anterior-Ischaemic optic neuropathy (AION) is manifested with painless total VA loss and swelling of the optic disc.

The review of literature describing effects of uncontrolled, fluctuated hypotension in development of AION is limited. We are predisposing that the fluctuation of BP could be triggering factor for development severe AION together with the duration of hypotension, but both of them is playing a major role in development of AION.

It remains poorly recognized as a complication by both renal physicians and ophthalmologists. We recommend intense control of Blood Pressure and ocular status in all patients undergoing Peritoneal Dialysis.

Uncontrolled hypotension can induced irreversible blindness in adult patients with peritoneal dialysis.

Keywords: Peritoneal Dialysis; Blindness; Hypotension; Ischaemic Optic Neuropathy

\section{Abbreviations}

AION: Anterior-Ischaemic Optic Neuropathy; VA: Visual Acuity; BP: Blood Pressure; NAAION: Non-Arteritic Anterior Ischemic Optic Neuropathy.

\section{Introduction}

Acute Bilateral blindness induced by AION is a rare eye complication for an adult patients undergoing peritoneal dialysis [1]. The data about bilateral, total blindness during or after peritoneal dialysis in adults patients is limited, We found only 3 cases of research papers where were described the cases of AION in peritoneal dialysis patients $[2,3]$.

Giacomo Di Zazzo., et al. reported Anterior ischemic optical neuropathy in children on chronic peritoneal dialysis: report of 7 cases, where was descried the increased risk of developing AION, which often results in irreversible blindness [1]. 
Stephanie Dufek 1 and colleagues reported Anterior ischemic optic neuropathy in pediatric peritoneal dialysis: risk factors and therapy, where they reported that Renal dialysis can render patients hypotensive, and this may be associated with anterior ischemic optic neuropathy. According the authors Therefore, 24-hour ambulatory blood pressure monitoring should be considered when investigating patients with suspected anterior ischemic optic neuropathy who are undergoing renal replacement [4].

Shveta Bansal., et al. and colleagues reported Hypotension-induced blindness in haemodialysis patients. According the authors Hypotension is a frequent observation and may provide the 'final insult' required to diminish flow to the optic nerve head resulting in often bilateral simultaneous visual loss [4].

A 51 -year-old female with end stage renal disease for previous 3 years, was receiving thrice weekly peritoneal dialysis. End Stage Renal disease with unknown etiology. She had a longstanding history of persistent systemic hypotension which was fluctuated $\sim 90 / 60$ to $60 / 30 \mathrm{mmHg}$, peripheral vascular disease, a previous episode of peritonitis.

She presented to our eye clinic with a sudden onset bilateral total loss of vision occurring soon after peritoneal dialysis. She was having a few episodes of total visual loss during last session of peritoneal dialysis (past 5 days). which she described as temporary episodes of blurred vision lasting approximately 4-5 min. Then the duration of losing of her vision was increasing, after last episode of peritoneal dialysis she lost her vision for 20-25 min. After last session of peritoneal dialysis she again had an episode of total vision loss and she was waiting to recover her vision but it did recovered. The next day patient was administrated to our department with binocular total blindness, IOP OD- $16 \mathrm{mmHg}$ OS-17 mmHg. Anterior segment without any kind pathologic changes, mild opacification of the lens. The vitreous was clear. Fundoscopy showed severe papilledema of both optic nerve head, accompanied by retinal vasculature changes, there was several hemorrhages close to the optic nerve head. Visual filed examination was not performed because of absence of visual functions.

On MRI of head and neck region there was not found any kind changes that could be triggering of development bilateral loss of vision.

She had found to have extremely low blood pressure in the few days preceding her loss of vision, measuring as low as 60/30
mmHg. She had long-standing fluctuating hypotension, worsened during each peritoneal dialysis treatment.

Her visual acuities were no perception of light in both eyes.

In addition there was no evidence of any intraocular inflammation or co-existent retinal pathology. This coupled with their swollen appearance and history of hypotension supported the diagnosis of anterior ischaemic optic neuropathy (AION) was made, and she was given pulsed iv $500 \mathrm{~m}$ g methylprednisolone per day for 2 days followed by oral prednisolone $250 \mathrm{mg}$ per day.

\section{Results and Discussion}

Information about the incidence, severity, and course of arterial hypotension in adults receiving chronic peritoneal dialysis (CPD) is limited. The largest case series so far was described by Malliara., et al. [1], who reported 81 hypotensive patients among 633 treated with CPD at 2 centers (Toronto, Ontario, Canada, and Thrace, Greece) between 1995 and 2000, for an incidence of 12.8\%. In those hypotensive patients, mean age was $63.8 \pm 14.2$ years and median duration of CPD was $49.3 \pm 30$ months.

C Basile., et al. and colleauges $\mathrm{C}$ reported that that most cases of AION occurring in chronic uremic patients on dialysis have some common features, including hypotension above all and anemia as common risk factors [5].

Dialysis-induced hypotension, the most frequent complication in patients treated with peritoneal dialysis.

Physicians must be aware of patients complaining of painless visual loss in this high-risk group, especially patients undergoing peritoneal dialysis treatment [4].

We advise careful monitoring of blood pressure to avoid sudden hypotensive episodes. AION occurring as a result of haemodialysisassociated hypotension was first reported by Groggel in 1986 [11].

Although there is yet much to be understood about the underlying mechanisms of AION and the exact role of blood pressure, the reported cases do share some common features.

There is a lot of questions about the underlying causes and physiology of AION in patients with peritoneal dialysis and the role of hypotension in formation of this condition. All reported cases have some common features. Mostly all reports is associated with the 
extremely low blood pressure during or after the dialysis $[2,4,10]$. Mostly in all cases there was visual loss episodes, before AION.

According Jackson., et al. blood pressure in a patient undergoing peritoneal dialysis who suffered from bilateral NAAION recorded diastolic readings as low as $41 \mathrm{mmHg}$ [2]. According Shveta Bansal., et al. Hypotension-induced blindness in haemodialysis patients they showed that low blood pressure recordings in their cases were as low as 70/50 $\mathrm{mmHg}$ [4].

In our case the BP of our patient after last episode of Peritoneal dialysis was $60 / 30$, and we are predisposing that the triggering factor form formation of AION in her case is fluctuation In the low BP. The definition of extremely low BP is individual for all patients, there is needed more data for understanding the mechanisms of formation AION in patients with peritoneal dialysis, and about the tolerated low blood Pressure before the formation of ischemic optic neuropathy.

The paper made the point that although blood pressure readings taken in a clinic setting had been normal, $24 \mathrm{~h}$ ambulatory readings showed significant dipping predisposing the patient to risk of AION [5-9].

\section{Conclusion}

The effects of uncontrolled especially fluctuated blood hypotension in development of AION is limited in literature. We predisposing that the fluctuation of Arterial BP could be more dangerous and triggering factor for development severe acute AION than the duration of hypotension, but both of them is playing a major role in formation of changes in optic nerve head. There is needed cooperation between treating nephrologist and ophthalmologists in all peritoneal dialysis patients, to prevent formation of ocular complications.

\section{Bibliography}

1. Di Zazzo G., et al. "Anterior ischemic optical neuropathy in children on chronic peritoneal dialysis: report of 7 cases". Dialysis International 35.2 (2015): 135-139.

2. T L Jackson., et al. "Hypotensive ischemic optic neuropathy and peritoneal dialysis". American Journal of Ophthalmology 128.1 (1999): 109-111.

3. Franz Schaefer., et al. "Peritoneal dialysis in infants: never lose sight of-and from-arterial hypotension!". Peritoneal Dialysis International 35.2 (2015): 123-124.
4. Shveta Bansal., et al. "Hypotension-induced blindness in haemodialysis patients”. Clinical Kidney Journal 7.4 (2014): 387390.

5. C Basile., et al. "Anterior ischemic optic neuropathy and dialysis: role of hypotension and anemia". Journal of Nephrology 14.5 (2001): 420-423.

6. Cuxart M., et al. "Acute bilateral visual loss in a haemodialysed patient". Nefrologia 25 (2005): 703-705.

7. Tay E., et al. "Anterior ischaemic optic neuropathy (AION) associated with post dialysis hypotension". Neuroophthalmologt 33 (2009): 168-173.

8. Nieto J and Zapata MA. "Bilateral anterior ischaemic optic neuropathy in patients on dialysis: a report of 2 cases". Indian Journal of Nephrology 20 (2010): 48-50.

9. Prat NM., et al. "Not just for men". Survey of Ophthalmology 56 (2011): 173-177.

10. Buthaina S. "Anterior ischaemic optic neuropathy and dialysis: effect of hypotension". Oman Journal of Ophthalmology 6 (2013): 64-65.

11. K S Servilla and G C Groggel. "Anterior ischemic optic neuropathy as a complication of hemodialysis". American Journal of Kidney Diseases 8.1 (1986): 61-63.

Volume 5 Issue 11 November 2021

(C) All rights are reserved by Ana Dvalishvili., et al. 